\title{
Aplicación de la técnica AHP para evaluar el efecto de los valores organizacionales en la productividad
}

\author{
Application of the AHP technique to evaluate the effect of organizational \\ values on productivity
}

\author{
Yngrid Naime Velasquez ', Miguel Núñez 2, Carlos Rodríguez Monroy ${ }^{3}$ \\ I Universidad Nacional Experimental de Guayana, UNEG - Venezuela. \\ 2 Dpto. Ingeniería Industrial. Universidad Nacional Experimental Politécnica, UNEXPO - Venezuela \\ 3 Escuela Técnica Superior de Ingenieros Industriales. Universidad Politécnica de Madrid. \\ ynaime@yahoo.es mnunezb@cantv.net crmonroy@etsii.upm.es
}

Fecha de recepción: 17-11-2009

Fecha de aceptación: 18-06-2010

Resumen: La Productividad es la relación integral entre personas, tecnología y dinero con el fin de generar bienes y servicios, que sean beneficiosos para la empresa, trabajadores, clientes y sociedad. El interés se centra en obtener una evaluación ponderada de los valores organizacionales asociados a los factores que afectan a la productividad a través del Proceso de Análisis Jerárquico (AHP). Del proceso de clasificación, ordenación y análisis se agruparon los factores internos que afectan la productividad en tres subsistemas: Cultura, Operaciones y Dirección. Los valores que obtuvieron la mayor preferencia fueron seguridad del trabajador, disciplina, ética y responsabilidad.

Palabras Clave: proceso de análisis jerárquico, productividad, valores organizacionales.

Abstract: Productivity is the integration of the variables, People, Technology and Money in order to generate goods and services which are beneficial to the company, employees, customers and society. The focus is on obtaining a balanced assessment of organizational values associated with factors that affect productivity through the Analytical Hierarchy Process (AHP). In the process of classification, management and analysis, the internal factors affecting productivity were grouped in three subsystems: Culture, Operations and Management. The values which obtained the highest ranking were worker safety, discipline, ethics and responsibility.

Key words: analytical hierarchy process, productivity, organizational values

\section{Introducción}

El entorno en el que están inmersas las organizaciones, caracterizado por clientes más exigentes y con mayor poder de elección, la globalización de los mercados, la innovación, el uso de tecnologías de información, la responsabilidad social, la gestión del conocimiento y la evaluación del medio ambiente, entre otros factores, obligan a las empresas a orientar esfuerzos en su gestión para hacer frente a una mayor presión competitiva, donde los términos productividad, calidad, oportunidad, velocidad, flexibilidad y costos son elementos indispensables para alcanzar el éxito.

En este orden de ideas, Lugo (2008), sostiene que la actividad de dirección de cualquier empresa u organización social, debe estar siempre en disposición de adaptarse al medio, a condiciones cambiantes a tra- vés de métodos, técnicas y estilos de dirección, en dependencia del nivel de consolidación del sistema organizacional de que se trate.

La gerencia es consciente de que hoy en día debe mejorar sus procesos, procurando alcanzar los niveles de productividad y calidad, que le permitan mantenerse y crecer en un mercado cada vez más exigente. Esta situación lleva a la gerencia a establecer mecanismos, normas y valores que permitan desarrollar en las personas el compromiso con la productividad.

Para incidir positivamente en la productividad, se hace necesario que las personas en la organización tengan sentido de pertenencia y aumenten su participación y compromiso con todos los procesos existentes en la organización. Hay que tener en cuenta que los esfuerzos por mejorar la productividad 
nunca serán suficientes ni estáticos, si consideramos la gran cantidad de variables que inciden en las operaciones de una organización.

Para Cardona, P. y Chinchilla (2008), las compañías que consiguen tener un alto rendimiento y que han sobrevivido a los cambios del entorno muestran una característica común como es la unidad, es decir empresa y trabajadores van juntos de la mano.

Andrew (2007), recomienda crear una cultura de rendimiento; considera que las organizaciones prósperas son las que tienen el rendimiento como parte de su cultura y enfocan la eficacia en las personas, ya que son el elemento clave para mejorar la productividad. En este sentido se hace necesario que sus valores estén alineados con la visión de la empresa.

Desde esta perspectiva, se considera que las personas deben tener conciencia de la importancia de la productividad para mejorar la calidad de vida y la organización, por su parte, debe valorar el rol insustituible de las personas para lograr incrementos en la productividad.

Se pone de manifiesto la importancia de los recursos humanos por ser los que llevan a cabo los procesos, tienen la responsabilidad de ejecutar y controlar las operaciones de manera que los resultados se correspondan con lo planificado. Si no se cuenta con personal capacitado, motivado y comprometido, difícilmente se podría producir lo que a corto, medio ó largo plazo afectará a la productividad y en consecuencia la competitividad de una organización.

En función de lo antes expuesto surge el presente estudio que tiene como objetivo obtener una evaluación ponderada de los valores organizacionales asociados a los factores que afectan a la productividad, utilizando el Proceso de Análisis Jerárquico (AHP). Para ello se identificaron los factores que afectan a la productividad y se consideraron los valores organizacionales como los elementos que impulsan las acciones de las personas en las organizaciones.

\section{Revisión de la literatura}

\section{I. Los valores organizacionales}

La Axiología (de axios, valor y logos, estudio o tratado) es la disciplina que estudia los valores. El método de la axiología es el análisis que permite encontrar los principios que deciden o permiten decidir fundamentalmente si algo es o no valioso: es decir, emitir un juicio, pero analizando los principios que permiten considerarlo valioso, (Pestana, 2004).

Hamburger (2008), define la axiología como una moderna disciplina filosófica que estudia los valores y se conoce con el nombre de filosofía de los valores. Cuando sale del ámbito estrictamente filosófico y se aplica a otros campos del saber como el psicológico o el empresarial, recibe el nombre de teoría de los valores. La palabra «valor» viene del verbo latino «valere» que significa estar bien, tener salud, vigor, fuerza, energía).

Escobar (2000), hace referencia a que los valores, en cuanto a directrices para la conducta, son los que dan a la vida humana, tanto individual como social, su sentido y finalidad, por lo que no puede concebirse una vida humana sin ideales, sin una tabla de valores que la apoye. Este planteamiento resulta de gran interés para entender la importancia de los valores en una organización, al ser los valores los que impulsan las acciones de las personas.

En esta investigación se hace mención especial al tema de los valores organizacionales, como elementos potenciadores para lograr que las personas se comprometan a alcanzar los objetivos de la organización, específicamente con el incremento de la productividad, cuyo resultado será beneficioso tanto para la empresa como para el trabajador.

De acuerdo a Napier y McDaniel (2006), a pesar de las diferencias entre los trabajadores, los estudios muestran que tienen valores comunes como son reconocimiento por el trabajo bien hecho, participación en las decisiones que afectan a su trabajo y un salario justo.

Estos resultados permiten inferir que en la medida en que se logren armonizar los valores de las personas con los de las organizaciones se obtendrán mejores beneficios para ambos.

\subsubsection{Definición de valores organizacionales}

Alcover et al. (2004), expresan que los valores constituyen el núcleo de la cultura organizacional y los definen como principios o creencias estructurados jerárquicamente, relativos a comportamientos o metas organizacionales deseables que orientan la vida de los miembros y están al servicio de intereses individuales, colectivos o mixtos. 
Según Hultman (2006), los valores son los conceptos psicológicos, internos a una persona. Expresa que las organizaciones no tienen valores, pero al estar conformadas por personas, sus culturas son expresiones de los valores existentes y son compartidos por ellas de distinta manera en la organización.

Koontz y Weihrich (2003) y Ogalla (2005), coinciden en que los valores guían las acciones y comportamientos de los empleados en el cumplimiento de los propósitos de la organización. La cultura está soportada en los valores, por lo tanto, en toda organización es posible observar si los valores que se proclaman se manifiestan en los modos de actuación, es decir, si existe relación entre lo que se dice y lo que realmente se hace.

García y Dolan (1997) definen los valores como aprendizajes estratégicos relativamente estables en el tiempo de que una forma de actuar es mejor que su opuesta para conseguir que salgan bien las cosas.

Hamburger (2008), resalta que los valores organizacionales establecen directrices para el compromiso diario, humanizan las relaciones en la empresa, dan sentido a la vida laboral, cohesionan los grupos, aumentan la producción y la calidad de los bienes y servicios, configuran la personalidad de la organización, generan confianza y credibilidad de los clientes en la empresa y fomentan el sentido de pertenencia de los empleados.

Los valores organizacionales, proporcionan un sentido de dirección común para todos los empleados y establecen directrices para su compromiso diario. Por ello es muy importante que una organización tenga sistematizados, compartidos e internalizados los valores que considera fundamentales para alcanzar el éxito.

\subsection{Factores que afectan a la productividad}

La productividad es la relación integral entre personas, tecnología y dinero con el fin de generar bienes y servicios, que sean beneficiosos para todos los actores involucrados: empresa, trabajadores, clientes y sociedad. La productividad permite a las organizaciones ser más competitivas, reducir sus costos de producción y aumentar su rentabilidad, lo cual coadyuva a un crecimiento sostenido en el mercado nacional e internacional, al enfrentarse con mayor éxito los retos de la globalización.
Cuando una organización tiene resultados negativos en la tasa de productividad, se observa su efecto en los precios al consumidor lo que generalmente desemboca en una disminución del volumen de las ventas, provoca desempleo y subutilización de la capacidad instalada.

Existen muchos factores que pueden afectar a la productividad de una organización; en relación a esto, lo fundamental es identificarlos y ver en qué medida la organización puede contrarrestar sus efectos negativos, así como también reforzar los de incidencia positiva, al diseñar o adoptar estrategias que impacten en los resultados.

En el Cuadro I de la página siguiente, se recogen algunos factores que afectan la productividad de acuerdo a distintos autores.

Según estos autores, hay factores sobre los cuales las empresas no tienen control, identificados como factores externos; en este sentido, se hace necesario diseñar estrategias que permitan enfrentarse a las situaciones adversas y aprovechar las oportunidades. Entre los factores externos se tienen: la política económica del gobierno, la administración pública, la infraestructura social, la estabilidad política, la competencia y la sociedad en su conjunto, entre otros.

En cuanto a los factores internos, dada su naturaleza de ser controlables por la empresa, son áreas de oportunidad para el mejoramiento de la productividad. Se convierten en elementos estratégicos, ya que las acciones o planes se deben diseñar bajo la premisa de que el mejoramiento de la productividad depende en gran medida de los efectos de la integración de los factores. Es importante determinar el grado de impacto que tienen en los resultados a fin de establecer medidas a corto, medo y largo plazo, que a su vez se traduzcan en acciones correctivas $y / 0$ preventivas.

Entre los factores internos se mencionan: ausentismo, rotación de personal, accidentes de trabajo, tiempo ocioso de los equipos, falta de materia prima, desperdicio de materia prima, obsolescencia de los equipos, calidad de la materia prima, fallos de los equipos, experiencia del trabajador, automatización de los procesos, métodos de trabajo, almacenamiento de la materia, disponibilidad de maquinarias y equipos, manejo de materiales, distribución de planta, incentivos por méritos al personal, diseño del producto, condición de las instalaciones, orden y limpieza del área de trabajo, capacitación del personal, distribución y asignación del personal, influencia sindical, pla- 
Cuadro I

Factores que afectan la productividad

\begin{tabular}{|c|c|}
\hline AUTOR & FACTORES QUE AFECTAN LA PRODUCTIVIDAD \\
\hline Bain, D. (1987) & $\begin{array}{l}\text { Los factores que restringen el incremento de la productividad son la incapacidad de la gerencia para pro- } \\
\text { piciar las condiciones para el mejoramiento, las reglamentaciones gubernamentales, el tamaño y la madu- } \\
\text { rez de las organizaciones, la falta de medición y evaluación de la productividad de la fuerza de trabajo y por } \\
\text { último los recursos físicos así como los factores tecnológicos. }\end{array}$ \\
\hline Sumanth, D. (1990) & $\begin{array}{l}\text { La inversión, la razón capital/trabajo, la investigación y desarrollo, la utilización de la capacidad, la reglamen- } \\
\text { tación del gobierno, la vida de la planta y el equipo, los costos de energía, la mezcla de la fuerza de trabajo, } \\
\text { la ética del trabajo, el temor de los empleados a perder su empleo, la influencia sindical y la administración. }\end{array}$ \\
\hline Schroeder, R. (1992) & $\begin{array}{l}\text { Hace mención a } 5 \text { factores, que comprenden factores externos, de capacidad e inventario, de producto, de } \\
\text { proceso, de fuerza de trabajo y de calidad, donde cada factor a su vez comprende varios aspectos y que pa- } \\
\text { ra mejorar la productividad hay que hacer la selección de la mezcla apropiada de estos factores para tomar } \\
\text { las acciones pertinentes. }\end{array}$ \\
\hline Prokopenko, J. (1999) & $\begin{array}{l}\text { Sostiene que existen factores internos y externos que propician el mejoramiento de la productividad. En- } \\
\text { tre los internos señala la tierra, la innovación tecnológica, las inversiones en nuevas plantas, la calidad de la } \\
\text { fuerza laboral, el mejoramiento de los sistemas, la aplicación de los principios de economía y alcance y la } \\
\text { habilidad de la administración para lograr la interrelación óptima de los factores que afectan la productivi- } \\
\text { dad. Entre los factores externos destaca la calidad de la política económica del gobierno, la administración } \\
\text { pública, la infraestructura social y de los negocios, la estabilidad política y los aspectos culturales, es decir la } \\
\text { sociedad como un todo. }\end{array}$ \\
\hline $\begin{array}{l}\text { Fernández, E., Avella, L. } \\
\text { y Fernández, M. (2003) }\end{array}$ & $\begin{array}{l}\text { Los factores que influyen en la productividad son de dos tipos: internos, que están sujetos al control de la } \\
\text { dirección de la empresa y externos, los cuales están totalmente fuera del ámbito de influencia de la direc- } \\
\text { ción de la empresa. }\end{array}$ \\
\hline Acevedo, D. (2004) & $\begin{array}{l}\text { Inversión, razón capital-trabajo, investigación y desarrollo, utilización de la capacidad, reglamentación del go- } \\
\text { bierno, vida útil de la planta y equipo, costo de energía, mezcla de la fuerza de trabajo, ética del trabajo, es- } \\
\text { tabilidad de los trabajadores, habilidades de los trabajadores, inteligencia y creatividad de los trabajadores, } \\
\text { influencia sindical, administración de los factores, el «orden y concierto» de los procesos, la lógica del es- } \\
\text { pacio, la tecnología, los sistema de información, el estilo de supervisión, la estructura organizativa y la cali- } \\
\text { dad de la gestión política. }\end{array}$ \\
\hline Ramírez, C. (2005) & El factor humano, el sindicalismo, la tecnología, el entorno político, el enfoque sistémico y la ergonomía. \\
\hline Anaya, J. (2006) & $\begin{array}{l}\text { Enumera } 4 \text { factores: la curva de aprendizaje, el diseño del producto, la mejora en los métodos de trabajo y } \\
\text { las mejoras técnicas. }\end{array}$ \\
\hline $\begin{array}{l}\text { Mosley, D., León, M., } \\
\text { y Pietri, P. (2005) }\end{array}$ & $\begin{array}{l}\text { Manifiestan que en las organizaciones la productividad se ve afectada por } 4 \text { grupos de factores que son: } \\
\text { I) La gerencia, la cual consideran de mayor importancia, por ser los responsables de impulsar los cambios } \\
\text { necesarios para lograr incrementos en la productividad; 2) El gobierno, que con sus disposiciones legales e } \\
\text { incentivos fiscales influyen de una manera u otra en la productividad de la organización. 3) Los trabajado- } \\
\text { res, porque su habilidad, motivación y compromiso repercute de una manera muy importante en el des- } \\
\text { empeño de las personas y los equipos de trabajo y 4) Los sindicatos, por el apoyo que dan en un momen- } \\
\text { to determinado a los cambios que se puedan presentar en la organización como son los avances tecnológicos, } \\
\text { los nuevos métodos de trabajo y en ocasiones la eliminación de puestos ineficientes. }\end{array}$ \\
\hline
\end{tabular}

Fuente: elaboración propia.

nificación del trabajo, disponibilidad de capital, crédito y salario, entre otros.

\section{Metodología}

Se realizó una investigación documental a fin de determinar los factores que afectan a la productividad. Para realizar este estudio, se aplicó la técnica de Proceso de Análisis Jerárquico (AHP), y se entrevistaron a representantes de la gerencia media y alta de una empresa metalmecánica del estado Bolívar en Venezuela, para obtener la evaluación de los valores organizaciones que afectan la productividad en dicha empresa.

\section{I. Proceso de Análisis Jerárquico (AHP)}

En las organizaciones, se presentan muchas situaciones en las que hay que tomar decisiones. Estas si- 
tuaciones deben ser analizadas por las personas que por su experiencia y conocimientos conocen todos los aspectos que las rodean. Esto facilita la comprensión del problema y la generación de alternativas de solución, que requieren para su selección la aplicación de métodos de Análisis de Decisión Multicriterio. (MCDA).

Rodríguez, S. (2008), plantea que los MCDA, permiten describir una colección de conceptos, métodos y técnicas que pretenden apoyar a los individuos o grupos a tomar decisiones que implican diferentes puntos de vista en conflicto y múltiples agentes interesados.

El Proceso de Análisis Jerárquico (AHP), desarrollado porThomas Saaty (200I), está diseñado para casos en los que las ideas, sentimientos y emociones se cuantifican sobre la base de juicios subjetivos para obtener una escala numérica y dar prioridades a las alternativas de decisión, permitiendo determinar la mejor solución ante un conjunto de alternativas, Taha (2004).

Esta técnica requiere que el tomador de las decisiones aporte juicios sobre la importancia relativa de cada objetivo y que a continuación especifique una preferencia sobre el objetivo de cada alternativa de decisión.
Según Berumen, y Llamazares (2007), el AHP se caracteriza por su flexibilidad, la cual facilita el entendimiento de la situación de los problemas. Esto permite llevar a cabo un proceso ordenado y gráfico de las etapas requeridas en la toma de decisiones. Asimismo, el AHP, permite analizar por separado la contribución de cada componente del modelo respecto al objetivo general.

\subsubsection{Etapas para el desarrollo del AHP}

- Definir el objetivo

El modelo consiste en estructurar un problema jerárquicamente en niveles. El primer nivel corresponde a la definición del problema. En esta etapa se desglosa el problema en sus componentes relevantes. La jerarquía básica está conformada por: meta u objetivo general, criterios y alternativas, (Martínez 2007).

En el Cuadro 2, se muestra la estructura del modelo jerárquico de decisiones AHP

- Establecer los criterios de evaluación

En esta etapa, el decisor emite sus juicios de valor o preferencias en cada uno de los niveles jerárquicos establecidos. Esta tarea consiste en una comparación

Cuadro 2

Modelo jerárquico de decisiones

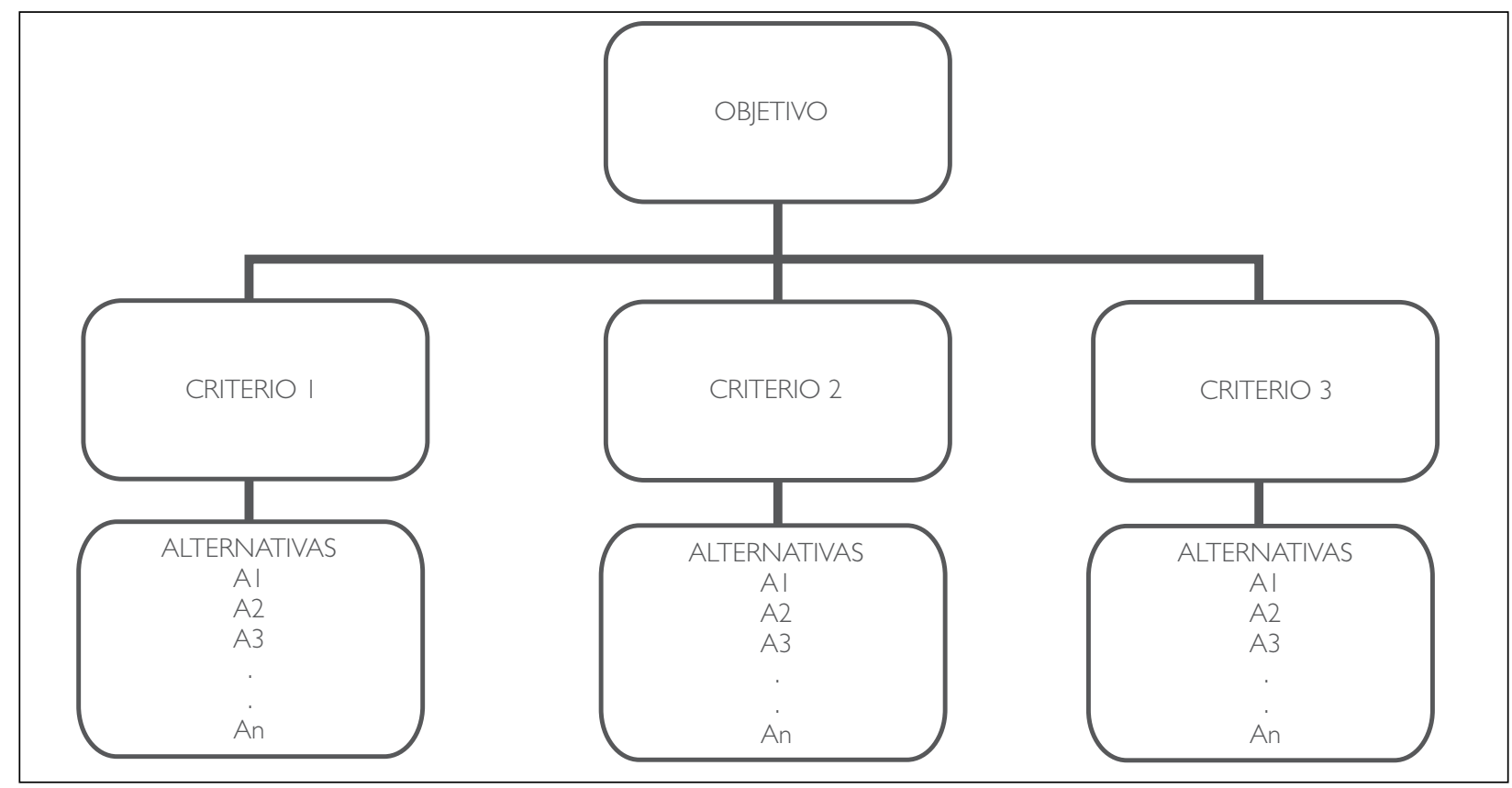

Fuente: elaboración propia. 
de valores subjetivos «por parejas» (comparaciones binarias); es decir, el decisor emite juicios de valor sobre la importancia relativa de los criterios y de las alternativas, quedando reflejada la dominación relativa, en términos de importancia, preferencia o probabilidad, de un elemento frente a otro, respecto de un atributo, o bien, si estamos en el último nivel de la jerarquía, de una propiedad o cualidad común, Martínez (2007).

El grado de importancia relativa o preferencia, se expresa a través de la escala de Thomas Saaty, ver Cuadro 3, donde se da el grado de importancia de cada criterio y a través de una matriz de comparación por pares se obtiene el peso de cada criterio.

Las decisiones en cuanto a la importancia relativa tendrán las siguientes expresiones: cuando dos elementos son igualmente importantes se asignará al par de elementos un I; moderadamente importante se representa por 3; fuertemente importante 5; mucho más importante 7 y extremadamente importante 9. Los números pares se utilizan para expresar situaciones intermedias, por ejemplo un elemento que se considere entre fuerte y muy fuerte tendrá una valoración de 8.

Martínez (2007), señala que la escala de Thomas Saaty, permite al decisor incorporar subjetividad, experiencia y conocimiento en un camino intuitivo y natural que está justificada teóricamente y cuya efectividad ha sido validada empíricamente aplicándola a diferentes situaciones.

- Establecer pesos a las alternativas
Consiste en establecer prioridades para cada alternativa en función de cada uno de los criterios, para lo cual se elabora una matriz de comparación por pares que contiene todas las alternativas y se evalúa de acuerdo a cada criterio.

Sea $a_{i j}$ el valor obtenido de la comparación del elemento $C_{i}$ con respecto al $C_{j}$. Suponiendo que el decisor es consistente en la emisión de juicios sobre cualquier par de elementos y sabiendo, además, que todos los elementos siempre tendrán una misma evaluación al compararse a sí mismos, se tiene que: $a_{i j} \cdot a_{j i}=1$ y $a_{i i}=1$. Por lo tanto solamente se deben efectuar $[\mathrm{l} / 2 \cdot \mathrm{m} \cdot(\mathrm{m}-\mathrm{I})]$ comparaciones para determinar el conjunto completo de juicios para los m elementos. Los datos correspondientes proporcionan una matriz recíproca de comparaciones pareadas $A_{(m \times m)}$. W. [Toncovich et al. (2007)]. En el Cuadro 4 de la página siguiente, se muestra la matriz normal $\mathrm{N}$, siendo el promedio de sus filas el peso de los criterios.

Una vez obtenida A, N y W, se determina la razón de consistencia RC:

$$
R C=\frac{1 C}{I A}
$$

Donde

$I C=\left(n_{\max }-n\right) /(n-I)=$ índice de consistencia de $A$

$I A=1,98(n-2) / n=$ índice de consistencia aleatoria de $A$

$n_{\max }=\sum_{i=1}^{n} \sum_{j=1}^{n} a_{i j} \bar{W}_{j}$

Cuadro 3

Escala de medidas de Saaty

\begin{tabular}{|c|l|l|}
\hline Escala numérica & \multicolumn{1}{|c|}{ Escala verbal } & \multicolumn{1}{|c|}{ Explicación } \\
\hline 1 & Igual importancia & $\begin{array}{l}\text { Los dos elementos contribuyen igualmente a la pro- } \\
\text { piedad o criterio }\end{array}$ \\
\hline 3 & $\begin{array}{l}\text { Moderadamente más importante un elemento que el } \\
\text { otro }\end{array}$ & $\begin{array}{l}\text { El juicio y la experiencia previa favorecen a un elemento } \\
\text { frente al otro }\end{array}$ \\
\hline 5 & Fuertemente más importante un elemento que el otro & $\begin{array}{l}\text { El juicio y la experiencia previa favorecen fuertemente } \\
\text { a un elemento frente al otro }\end{array}$ \\
\hline 7 & $\begin{array}{l}\text { Mucho más fuerte la importancia de un elemento que } \\
\text { la del otro }\end{array}$ & $\begin{array}{l}\text { Un elemento domina fuertemente. Su dominación es- } \\
\text { tá probada en práctica }\end{array}$ \\
\hline 9 & Importancia extrema de un elemento frente al otro & $\begin{array}{l}\text { Un elemento domina al otro con el mayor orden de } \\
\text { magnitud posible }\end{array}$ \\
\hline
\end{tabular}

Fuente: Martínez (2007). 
Cuadro 4

\section{Matriz recíproca de comparaciones pareadas}

\begin{tabular}{|c|c|c|c|c|c|c|c|c|c|}
\hline \multirow{5}{*}{$A=$} & & & & \multirow{5}{*}{$\rightarrow N=$} & \multirow{5}{*}{$\begin{array}{l}a_{1 \mid} / \sum a_{\mathrm{ml}} \\
a_{2 \mid} / \sum a_{\mathrm{m} \mid} \\
a_{\mathrm{m}} / \sum a_{\mathrm{m}}\end{array}$} & \multirow{4}{*}{$\begin{array}{l}a_{12} / \Sigma a_{m 2} \\
a_{22} / \Sigma a_{m 2} \\
a_{m 2} / \Sigma a_{m 2}\end{array}$} & \multirow{4}{*}{$\begin{array}{l}a_{1 \mathrm{~m}} / \Sigma a_{m m} \\
a_{2 \mathrm{~m}} / \Sigma a_{m m} \\
a_{\mathrm{mm}} / \Sigma a_{m m}\end{array}$} & \multirow{4}{*}{$\rightarrow$} & $\bar{W}_{i}$ \\
\hline & $a_{11}$ & $a_{12}$ & $a_{1 m}$ & & & & & & $\bar{W}_{1}$ \\
\hline & $a_{21}$ & $a_{22}$ & $a_{2 m}$ & & & & & & $\bar{W}_{2}$ \\
\hline & $a_{\mathrm{ml}}$ & $a_{m 2}$ & $a_{m m}$ & & & & & & $\bar{W}_{m}$ \\
\hline & $\sum a_{m \mid}$ & $\Sigma a_{m 2}$ & $\Sigma a_{m m}$ & & & & & & I \\
\hline
\end{tabular}

Los valores de RC menores de 0.10 indican juicios consistentes.

- Toma de decisión:

Obtenido el grado de importancia de cada alternativa con respecto a cada criterio, se construye una matriz con estas preferencias y los pesos de cada criterio. La decisión se produce al sumar el producto de multiplicar el peso de cada alternativa por el peso de cada criterio. La decisión se toma al evaluar los resultados de cada alternativa con respecto a cada criterio.

La estructura jerárquica del problema de decisión en la presente investigación comprende:

Nivel I: se intenta establecer en qué medida los valores organizacionales afectan la productividad.

Nivel 2: los criterios considerados, son los subsistemas cultura, operaciones y dirección, en los cuales se han agrupado solamente los factores internos que afectan a la productividad, excluyéndose en esta investigación los factores externos, por no tener la organización influencia directa sobre estos factores.

Nivel 3: corresponde a los valores organizacionales que se consideran asociados a los factores internos que afectan a la productividad.

\section{Resultados}

De acuerdo a los factores que afectan a la productividad, identificados en la revisión bibliográfica, se observa que pertenecen o dependen básicamente de dos elementos: operaciones y dirección. Sin embargo, hay un tercer elemento a considerar, que si bien es cierto que no está claramente identificado en los factores encontrados, no es menos importante como es el elemento cultural. Éste es base fundamental de cualquier organización, por ser el engranaje entre las operaciones y la dirección.
Basándonos en O'Donnell (2007), el ser humano es el principio, el medio y el fin de cualquier proceso de transformación, de allí su influencia en los resultados que se obtienen en los procesos de producción.

Lo antes expuesto permite hacer comparaciones y agrupar los factores internos que afectan a la productividad, obteniéndose una clasificación que abarca tres subsistemas: Cultura, Dirección y Operaciones.

El subsistema Cultura contempla las normas, creencias, valores que pueden estar implícitos o explícitos en una organización y son los que rigen el modo de actuación de las personas, lo cual es tomado como una orientación para actuar en la organización, sin menoscabar la libertad individual.

El subsistema Dirección, es el responsable de armonizar al personal, la tecnología y el dinero. Su principal rol es liderar los procesos, para aprovechar al máximo y de manera eficiente todos los recursos.

El subsistema Operaciones, contempla las actividades necesarias para producir de acuerdo a las especificaciones, involucra a las personas, materiales, equipos, métodos y capital. El gran reto consiste en su combinación y utilización óptima. (Ver Cuadro 5).

\section{Cuadro 5}

Factores internos que afectan a la productividad

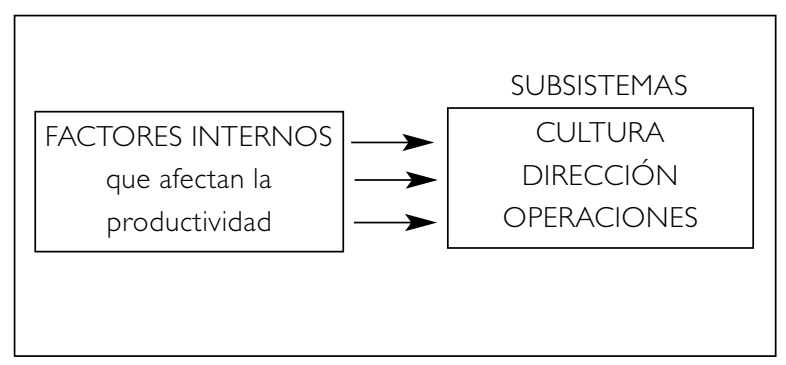

Fuente: Elaboración Propia 
El Proceso de Análisis Jerárquico, muestra que el criterio más importante es la Cultura, los dos criterios restantes tienen igual grado de importancia y sus pesos fueron obtenidos a través de la matriz de comparaciones, como se muestra en el Cuadro 6.

La Razón de Consistencia (RC), viene determinada por.

$$
\begin{gathered}
R C=\frac{I C}{I A}=\frac{\left(n_{\max }-n\right) I_{n-1}}{1,98(n-2) /_{n}} \\
n_{\max }=\sum_{i=1}^{n} \sum_{j=1}^{n} a_{i j} \bar{w}_{j}\left|\begin{array}{lll}
1.00 & 3.00 & 3.48 \\
0.30 & 1.00 & 1.27 \\
0.22 & 1.45 & 1.00
\end{array}\right| \quad\left|\begin{array}{l}
0.60 \\
0.20 \\
0.20
\end{array}\right|=\left|\begin{array}{l}
1.8845 \\
0.6256 \\
0.6208
\end{array}\right|
\end{gathered}
$$

$R C=\frac{I C}{I A}=\frac{(3,1310-3) / 3-1}{1,98(3-2) / 3}=0,0992, R C$ me-

nor que 0,1 , por lo tanto hay consistencia.

En la Figura I, se muestra la estructura del AHP, con las respectivas importancias relativas de las alternativas sobre cada uno de de los criterios, las cuales fueron obtenidas a través de una matriz de comparación por pares normalizada.

En la matriz de resultados, ver Cuadro 7 de la página siguiente, se muestra el resultado de la evaluación que se obtiene al multiplicar el peso de cada criterio por el desempeño de cada alternativa.

Cuadro 6

\begin{tabular}{|c|c|c|c|c|c|c|c|c|c|}
\hline & & & & & & & & & $\bar{W}_{i}$ \\
\hline \multirow{4}{*}{$A=$} & 1.00 & 3.00 & 3.48 & & 0.658 & 0.550 & 0.606 & & 0.60 \\
\hline & 0.30 & 1.00 & 1.27 & $\rightarrow N=$ & 0.194 & 0.184 & 0.220 & $\rightarrow$ & 0.20 \\
\hline & 0.22 & 1.45 & 1.00 & & 0.147 & 0.267 & 0.174 & & 0.20 \\
\hline & 1.52 & 5.45 & 5.75 & & 1.000 & 1.000 & 1.000 & & 1.00 \\
\hline
\end{tabular}

Matriz recíproca de comparaciones pareadas

Figura I

Estructura jerárquica del AHP

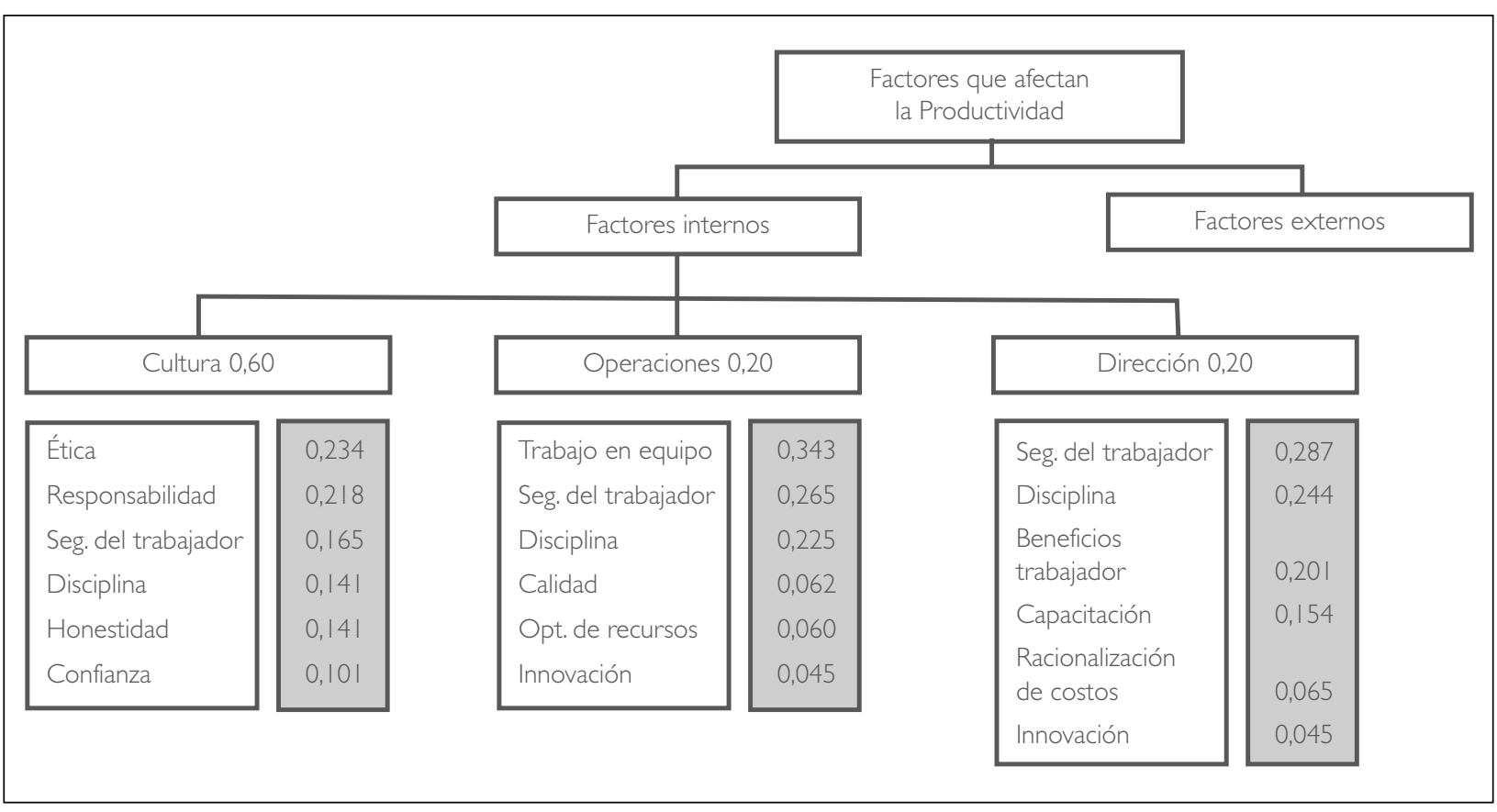


Se observa que el efecto de los valores en los factores que afectan la productividad está dado por la ética, trabajo en equipo, seguridad del trabajador, beneficios del trabajador, disciplina, responsabilidad, capacitación, confianza y honestidad.

De acuerdo a la matriz de resultados, los valores disciplina y seguridad del trabajador, son los únicos valores que fueron considerados en los tres subsistemas, por lo que deben ser vistos en la organización como valores claves para el seguimiento del comportamiento de la productividad.

\section{Conclusiones}

El Proceso de Análisis Jerárquico (AHP) permite a la gerencia establecer el grado de importancia que tienen los valores organizacionales asociados a los factores que afectan a la productividad en su organización.

Se observa que los valores organizacionales como elementos de la cultura sirven de guía a los miembros de las organizaciones para realizar sus tareas y su puesta en práctica coadyuva a la transparencia en todos los procesos que fortalecen el buen desempeño de la organización, satisfaciendo intereses individuales y colectivos, tanto de la organización como de la sociedad.
Los resultados de la presente investigación, permiten identificar tres subsistemas que agrupan los factores internos que afectan a la productividad, denominados: Cultura, Operaciones y Dirección.

La aplicación de la técnica de Proceso de Análisis Jerárquico (AHP), permitió conocer la opinión de la Gerencia sobre los valores que afectan a la productividad. De acuerdo a esta evaluación, los valores que tienen mayor impacto en la productividad son: seguridad del trabajador, disciplina, ética y responsabilidad. Los valores seguridad del trabajador y disciplina tienen efecto en los tres subsistemas definidos en esta investigación. En este sentido, deben ser los prioritarios a incluir en el proceso de evaluación del crecimiento de la productividad.

Finalmente, se considera pertinente ampliar la validación de este sistema de valores en empresas de distintos sectores a fin de de lograr un sistema lo más aproximado a las organizaciones, independientemente del sector al que pertenecen para posteriormente determinar la operacionalización de cada valor para medir su efecto en la productividad.

\section{Referencias}

ACEVEDO, D. (2004). Metodología del control de gestión: Del indicador a la toma de decisiones. Venezuela: Ediciones Dibrant Publicidad de Venezuela.

Cuadro 7

Matriz de resultados

\begin{tabular}{|l|c|c|c|c|c|c|c|c|}
\hline \multirow{2}{*}{ Alternativas } & \multicolumn{3}{c|}{$\begin{array}{c}\text { Puntuación delValor } \\
\text { Organizacional en el contexto }\end{array}$} & \multicolumn{3}{c|}{ Criterios } & \multicolumn{2}{c|}{ Resultado } \\
\hline & Cultura & Operaciones & Dirección & Cultura & Operación & Dirección & \multirow{2}{*}{ R= CV+O+D } & $\%$ acumulado \\
\hline \multicolumn{1}{|c|}{ Alternativas } & A & B & C & CV=A*0.60 & O=B*0.2 & D=C*0.20 & IR \\
\hline Seguridad del trabajador & 0,1650 & 0,2650 & 0,2870 & 0,0990 & 0,0530 & 0,0570 & 0,2090 & $20,90 \%$ \\
\hline Disciplina & 0,1410 & 0,2250 & 0,2440 & 0,0850 & 0,0450 & 0,0490 & 0,1780 & $38,70 \%$ \\
\hline Ética & 0,2340 & & & 0,1400 & & & 0,1400 & $52,70 \%$ \\
\hline Responsabilidad & 0,2180 & & & 0,1310 & & & 0,1310 & $65,80 \%$ \\
\hline Honestidad & 0,1410 & & & 0,0850 & & & 0,0850 & $74,30 \%$ \\
\hline Trabajo en equipo & & 0,3430 & & & 0,0690 & & 0,0690 & $81,20 \%$ \\
\hline Confianza & 0,1010 & & & 0,0610 & & & 0,0610 & $87,30 \%$ \\
\hline Beneficios del trabajador & & & 0,2010 & & & 0,0400 & 0,0400 & $91,30 \%$ \\
\hline Capacitación & & & 0,1540 & & & 0,0310 & 0,0310 & $94,40 \%$ \\
\hline Innovación & & 0,0450 & 0,0450 & 0,0000 & 0,0090 & 0,0090 & 0,0180 & $96,20 \%$ \\
\hline Racionalización de costos & & & 0,0650 & & & 0,0130 & 0,0130 & $97,50 \%$ \\
\hline Calidad & & 0,0620 & & & 0,0120 & & 0,0120 & $98,70 \%$ \\
\hline Optimización de recursos & & 0,0600 & & & 0,0120 & & 0,0120 & $99,90 \%$ \\
\hline
\end{tabular}

Fuente: elaboración propia 
ALCOVER, C., MARTíNEZ, I. (2004). Introducción a la psicología del trabajo. España: McGraw-Hill.

ANAYA, J. (2006). Logística integral. La gestión operativa de la empresa. España: Editorial ESIC

ANDREW, E. (2007). How do you measure productivity? CA Magazine, I40(6), 12.

BAIN, D. (1987). Productividad. México: McGraw-Hill.

BERUMEN, S. y LLAMAZARES, F. (2007). La utilidad de los métodos de decisión multicriterio (como el AHP) en un entorno de competitividad creciente. Cuadernos de Administración, 20(34), 65-87.

CARDONA, P. y CHINCHILLA, M. (2008). Estrategia en el siglo XXI. España: Deusto.

ESCOBAR, G. (2000). Ética. México: McGraw Hill.

FERNÁNDEZ, E., AVELLA, L. y FERNÁNDEZ, M. (2003). Estrategia de producción. España: Mc Graw Hill.

GARCÍA, S., y DOLAN, S. ( 1 997). La dirección por valores. España: McGraw Hill.

HAMBURGER, A.(2008).Los valores corporativos en la empresa.Colombia: Paulinas.

HULTMAN, K.(2005). Evaluating organizational values.Organization Development Journal, 23(4).

KOONTZ, H., y WEIHRICH, H. (2003). Administración. Una perspectiva global. México: Mc Graw Hill.

LUGO, R. (2008). La dirección por objetivos y valores con enfoque estratégico. Teoría y práctica empresarial en CIMEX. Retrieved Mayo/5, 2009, from http://ideas.repec.org/a/ erv/observ/y2008il05 I4.htm

MARTÍNEZ, E. (2007). Aplicación del proceso de análisis jerárquico en la selección de la localización de una PYME. Anuario Jurídico y Económico Escurialense, (40), 523.

MOSLEY, D. LEÓN, M. y PIETRI, P. (2005). Supervisión. La práctica del empowerment, desarrollo de equipos de trabajo y su motivació. México:THOMSON
NAPIER, R., y MCDANIEL, R. (2006). Measuring what matters. USA: Davies-Black

O'DONNELL, K. (2007). Valores humanos en la empresa. España: LID Editorial Empresarial

OGALLA, F. (2005). Sistema de gestión. España: Diaz de Santos.

PESTANA, P. (2004). Aproximación conceptual al mundo de los valores. REICE-Revista Electrónica Iberoamericana sobre Calidad, Eficacia y Cambio en Educación, 2(2)

PROKOPENKO, J. (1999). Organizaciones Promotoras de Productividad. Evolución y Experiencia. Organización Internacional del Trabajo. Departamento de desarrollo cooperativo y empresa. Programa de desarrollo organizativo y productividad.

RAMÍREZ, C. (2005). Administrando la calidad para el cambio. México: LIMUSA.

RODRÍGUEZ, S. (2008). Toma de decisión multicriterio con AHP, ANP y Lógica Difusa. Maestría en Ingeniería de Sistemas y Computación. Universidad Nacional de Colombia. Retrieved junio/20, 2008. http://scholar.google.co.ve/scholar?hl=es\&q=Toma + de ++ decision + multic riteria+con+AHP\% 2C + ANP + y + L\% C3\% B3gica + Difusa \& btnG $=$ Buscar \& Ir $=$ \& as_ylo $=\&$ as_vis $=0$

SAATY,T. (200I). Fundamentals of Decision Making and Priority Theory. Pittsburgh, Pennsylvania: RWS Publications. ISBN 0-96203|7-6-3.

SCHROEDER, R. (1994).Administración de operaciones. México: Mc Graw-Hill.

SUMANTH, D. (1990). Ingeniería y administración de la productividad. México: Mc Graw-Hill.

TAHA, H. (2004). Investigación de operaciones. México: Pearson.

TONCOVICH, A., MORENO-JIMÉNEZ, J. y CORRAL, R. (2007). Selección multicriterio de un sistema ERP mediante las metodologías AHPYANP. Retrieved Octubre/ I 3, 2009, from http://www.cnc-logistica.org/congreso-cnc/documentos/80.pdf 